\title{
EDITORIAL
}

\section{From bedlam to bedroom: almost there?}

\section{Journal of Perinatology (2010) 30, 303-304; doi:10.1038/p. 2010.45}

Science has promised us truth. It has never promised us either peace or happiness.

Gustave Le Bon

As the science of neonatology advanced over the latter part of the 20th century, neonatal intensive care units (NICUs) did become happier places, with fewer codes and deaths. But they were hardly peaceful, becoming busier, brighter, noisier and far more complex. With the risk of death receding for most babies, concern over the effect of these stressors on infant neurodevelopment, the NICU experience and satisfaction of their families, and the physical and emotional health of their caregivers has grown. One reaction to this concern has been the recent surge in construction of NICUs with most or all of their bed spaces in single-family rooms (SFRs).

That SFRs may be better for babies directly by reducing exposure to noxious sensory stimuli and the risk of nosocomial infection, and indirectly by increasing skin-to-skin care through encouraging parental presence and intimacy are attractive hypotheses. Studies on adults in private room settings have demonstrated benefits, ${ }^{1}$ but randomized controlled trials of these questions in newborns are difficult to undertake when the intervention involves construction of a multi-million dollar facility. Alternative strategies include the use of historical controls, or, in cases where 'hybrid' units exist (which combine both private and multi-bed rooms), concurrent controls, although these methods have obvious shortcomings.

Assessing the satisfaction of parents and caregivers after moving from an 'open' NICU to an SFR NICU is a more accessible question. A previous study ${ }^{2}$ as well as a large body of anecdotal information has demonstrated that families are more satisfied in private rooms, but are challenged by the isolation this setting imposes upon them. Caregivers, too, recognize that an SFR design improves the sensory environment for newborns and is more desirable for families, but creates communication and collaboration impediments for both families and staff that are not found in open, multi-bed rooms. ${ }^{3-5}$

In this issue, two studies using historical controls of multi-bed NICUs showed that moving to a new SFR NICU may improve staff perception of workplace quality (Stevens et al. ${ }^{6}$ ), but this is dependent to some degree on the type of caregiver and the length of time after the move during which the caregivers are surveyed (Domanico et al. ${ }^{7}$ ). Furthermore, the Domanico study showed that caregiver concerns must be considered subjective, as the perception that the safety of the infant might be compromised in the SFR could not be confirmed by increases in mortality or length of stay.

The Domanico study also evaluated parental satisfaction and demonstrated that, similar to caregivers, the heterogeneity of this population leads to differing conclusions about the advantages and disadvantages of SFR design. Parents who had experience in both units tended to favor the SFR NICU, and parents with longer lengths of stay also preferred the SFR. Even so, open units were still considered better for promoting interaction with other families.

What conclusions can be drawn from studies that are clearly subjective and rely on historical controls? First, there can be little doubt that the relative isolation experienced in SFR NICUs by both families and staff is a real and important challenge. Strategies to address this concern require both design and operational features not always anticipated or incorporated into new SFR NICUs. Designs should provide gathering spaces within the patientcare areas for both caregivers and families that are convenient, welcoming and have views to the outside world, as well as easy visibility to the patient rooms. Operationally, staff must be provided with communication devices that allow them to know what's going on with multiple babies and with their colleagues, and that keep them in touch with other support services in the hospital and accessible to calls from outside. Programs should also be available for families that give them a reason to gather for meals, education, mutual support and relaxation. It will be apparent that although these design and operational features are essential in an SFR NICU, they are valuable regardless of the general design chosen.

The second conclusion the current studies reinforce is that families are appreciative of a private space to share with their infant, especially if the length of stay is for more than a few days. This is a basic right (codified by HIPAA), but it also has potential benefit for the infant-both the Domanico study and an earlier study from the Vanderbilt group ${ }^{2}$ anecdotally reported increased parental presence in the SFR unit. Moreover, a recent study from the Karolinska group ${ }^{8}$ showed that full-time parental presence led to reduced length of stay and chronic lung disease for their babies when compared with those whose parents visited for several hours a day but did not have access to overnight accommodations in their baby's room.

A third conclusion drawn from the current studies as well as from extensive anecdotal evidence is that although caregivers find challenges in the SFR environment, few of them believe that the conventional open-bay design of the 1980s and 90s is better for their patients. This extends an observation that has held true 
throughout the history of the NICU — and medicine in general - that caregivers often sacrifice their own needs to those of their patients. It is our challenge to design and operate environments that do no ask them to make those sacrifices any more than absolutely necessary.

The concept of the NICU as a peaceful, nurturing place would have been considered quixotic a generation ago; it now seems to be a realistic target, yet challenges remain. Further progress will require attention to the many remaining stressors, both physical (for example, pain and other noxious stimuli) and mental (for example, poor communication) that we may still consider inherent and inescapable. Indeed, some do exist in tension with one another; reducing a particular stress on babies may put additional stress on caregivers. As in the past, answers will probably derive from both design and operational advances, ones that anyone designing a new unit should try to anticipate and imbed into their visioning and planning process. A good NICU design requires familiarity not only with neonatal medicine, but also with sociology, psychology, anthropology and occupational health - indeed, with all the allied sciences that inform us about how best to live and work together as a community.

\section{Conflict of interest}

The authors declare no conflict of interest.
RD White

Newborn ICU, Memorial Hospital, South Bend, IN, USA E-mail:Robert_Wbite@pediatrix.com

\section{References}

1 Berry LL, Parker D, Coile Jr RC, Hamilton DK, O'Neill DD, Sadler BL. The business case for better buildings. Healthc Financ Manage 2004; 58: 76-84.

2 Carter BS, Carter A, Bennett S. Families' views upon experiencing change in the neonatal intensive care unit environment: from the 'baby barn' to the private room. J Perinatol 2008; 28: 827-829.

3 Walsh WF, McCullough KL, White RD. Room for improvement: nurses' perceptions of providing care in a single room newborn intensive care setting. Adv Neonatal Care 2006; 6: 261-270.

4 Harris DD, Shepley MM, White RD, Kolberg JHS, Harrell JW. The impact of single family room design on patients and caregivers: executive summary. J Perinatol 2006; 26 S38-S48.

5 Shepley MM, Harris DD, White R. Open-bay and single-family room neonatal intensive care units: caregiver satisfaction and stress. Environ Behav 2008; 40: 249-268.

6 Stevens DC, Helseth CC, Khan MA, Munson DP, Smith TJ. Neonatal intensive care nursery staff perceive enhanced workplace quality with the single-family room design. J Perinatol 2010; 30: 354-361.

7 Domanico R, Davis DK, Coleman F, Davis BO. Documenting the NICU design dilemma: parent and staff perceptions of open ward versus single family room units. J Perinatol 2010; 30: 343-353.

8 Ortenstrand A, Westrup B, Brostrom EB, Sarman I, Akerstrom S, Brune T et al. The Stockholm neonatal family centered care study: effects on length of stay and infant morbidity. Pediatrics 2010; 125: e278-e285. 